\title{
Estimation de la diffusion effective dans un écoulement instationnaire
}

\author{
${ }^{(a)}$ Lahcen Aitmoudid, ${ }^{\text {(b) }}$ Abdellatif Ouahsine, ${ }^{(\mathrm{c})}$ M.Taki, ${ }^{(\mathrm{d})}$ K.Korotenko \\ (a) \\ Doctorant, Centre de Calcul et de Modélisation de Lens, \\ Faculté des Sciences Jean Perrin, F-62307 Lens Cedex. \\ Professeur, Laboratoire Roberval, UMR-CNRS 6066, Université de Technologie de Compiègne, \\ B.P. 20529, 60205 Compiègne Cedex. \\ (c) \\ Chercheur, Laboratoire des pectroscopie Hertzienne, Université de Lille I, \\ Chercheur, Institut P.P Shirshov d'Oceanologie, Moscow, Russie. \\ ouahsine@utc.fr
}

\section{Résumé}

Un modèle lagrangien pour étudier le transport d'une concentration ou d'un traceur passif dans un écoulement instationnaire est proposé. Il est basé sur une approche stochastique de simulation des trajectoires des particules pour estimer la diffusion effective du traceur en tenant compte de la turbulence et de la topologie du milieu. L'objectif est de caractériser le rôle de la diffusion moléculaire dans ce processus de transport. Dans le cas stationnaire, lorsque le coefficient de diffusion moléculaire est nul, la diffusion de la concentration dépend des lignes des courants, mais elle ne l'est pas lorsque ce coefficient est petit. Dans le cas instationnaire le chaos peut intervenir et jouer un rôle important dans la dispersion des particules.

\begin{abstract}
$\underline{\text { Abstract }}$
A Lagrangian model to predict the transport of a tracer concentration in a transient flow is presented. It is based on the particle trajectory by taking into account the turbulence and the domain topology. The main goal is to show how them olecular diffusion coefficient is related to the nature of the flow. In the stationary flow case, when this coefficient is null, the diffusion of the concentration depends on the flow stream lines, but when it's small but non zero the concentration does not depend on the flow stream lines. In the transient flow case the chaos can take place and have an important role in the particle dispersion process.
\end{abstract}

Mots-clés: Transport lagrangien; diffusion effective; suivi de particules.

\section{Introduction}

Dans ce travail, ont été examiné les trajectoires lagrangiennes d'un nuage de particules en considérant l'équation de Langevin dans le cas bidimensionnel. Pour tenir compte des fluctuations des vitesses dues à la turbulence et à d'autres perturbations, un bruit blanc, considéré ici comme Brownien, a été ajouté à cette équation. A partir d'un champ de vitesse eulerien donné, ces trajectoires sont déterminées et analysées en fonction du coefficient de diffusion moléculaire et également en fonction de la nature de l'écoulement: stationnaire ou instationnaire.

Deux cas d'applications sont considérés. (i) Le premier cas correspond à un cas test académique où l'on a considéré un champs de vitesse instationnaire donné. Les particules sont initialement lâchées et advectées par ce champ selon deux configurations; la première 
configuration correspond aux lignes de courant fermées et la deuxième aux lignes de courant ouvertes. (ii) Le deuxième cas est une application à la Manche orientale pour illustrer la diffusion et la dispersion des concentrations dans un cas réel.

\section{$\underline{\text { 2.Formulation du problème }}$}

\subsection{Equation d'advection - diffusion}

On considère l'équation sans dimension d'advection-diffusion d'une concentration passive suivante:

$$
S_{t} \frac{\partial \Phi}{\partial t}+\mathbf{u} \nabla \Phi=\frac{1}{P_{e}} \Delta \Phi
$$

Où $\mathrm{St}=\mathrm{L} / \mathrm{UT}$ désigne le nombre de Strouhal qui caractérise la stationnarité de l'écoulement, avec L, U et T désignent respectivement des grandeurs caractéristiques de longueur, de vitesse et du temps; désigne la concentration, $\mathrm{u}(\mathrm{x}, \mathrm{t})$ est le champ des vitesses euleriennes. Le fluide considéré est incompressible et vérifie $\nabla . \mathrm{u}=0$; la relation qui existe entre la fonction Hamiltonienne $^{\psi=\psi(x, t)}$ et le champs u vérifie:

$$
u=\frac{\partial \psi}{\partial y} ; \quad v=-\frac{\partial \psi}{\partial x}
$$

e=UL/Dmol désigne le nombre de Péclet où Dmol est le coefficient de diffusion moléculaire. Pour mettre en évidence l'influence de cette diffusion moléculaire, on examine le comportement de la solution de (1) en fonction du coefficient Dmol suivants deux cas:

\subsection{1.cas stationnaire: $\mathrm{St}=0()<1$,}

1. si Dmol =0, de l'équation (1) on obtient:

$$
J(\phi, \psi)=-\frac{\partial \psi}{\partial y} \frac{\partial \Phi}{\partial x}+\frac{\partial \psi}{\partial x} \frac{\partial \Phi}{\partial y}=0
$$

où $\mathrm{J}$ désigne le jacobien. Des équations (1) et (3) on déduit que la variation: $d \Phi=\frac{\partial \psi}{\partial x} \frac{\partial \Phi}{\partial \psi} d x+\frac{\partial \psi}{\partial y} \frac{\partial \Phi}{\partial \psi} d y$ est non nulle, et donc:

$$
\Phi=\Phi(\psi)
$$

ce qui montre que dépend des ignes de courant, et que la diffusion dépend de la structure de l'écoulement.

2. si Dmol $=0()<<1$, on considère le développement asymptotique: $=0+1+\mathrm{O}\left({ }^{2}\right)$.

A l'ordre zéro en , l'équation (1) s'écrit:

$$
J\left(\Phi_{1}, \psi\right)=\left(\frac{\partial^{2}}{\partial x^{2}}+\frac{\partial^{2}}{\partial y^{2}}\right) \Phi_{0}
$$


En supposant qu'il existe un domaine D fermé, délimité par les lignes de courant, on a:

$$
\iint_{D} J\left(\Phi_{1}, \psi\right) d x d x=\iint_{D}\left(\frac{\partial^{2}}{\partial x^{2}}+\frac{\partial^{2}}{\partial y^{2}}\right) \Phi_{0} d x d y
$$

En vertu de l'équation (3), le terme de gauche s'annule. Ensuite, en utilisant le théorème de Green- Riemann appliqué aux intégrales curvilignes, le terme de droite de l'équation (6) peut être exprimé sous forme d'une intégrale curviligne, il vient:

$$
\oint_{c} \frac{d \Phi_{0}}{d \psi} d s=0
$$

On a: $\frac{d \Phi_{0}}{d \psi}=0$, il s'en suit alors que 0 ne dépend plus de . Ce dernier constat, permet de conclure que lorsque le coefficient de diffusion moléculaire Dmol est faible et non nul, la diffusion de la concentration peut être influencée par d'autres processus, comme la turbulence, le chaos, etc.

\section{$\underline{\text { 3.Processusdetransportdanslecasinstationnaire }}$}

Dans le cas instationnaire on suppose que $\mathrm{St}=0$ (1), la solution de l'équation (1) s'écrit:

$$
\Phi(x, t)=\frac{1}{\sqrt{4 \pi D_{\text {mol }} t}} \exp \left[-\frac{(x-u t)^{2}}{4 D_{\text {mol }} t}\right]
$$

Sachant que la moyenne des positions hxi est nulle, c'est à dire que:

$$
\langle x\rangle=\int_{-\infty}^{+\infty} x \phi(x, t) d x=\frac{1}{\sqrt{4 \pi D_{\text {mol }}}} \int_{-\infty}^{+\infty} x \exp \left[-\frac{(x-u t)^{2}}{4 D_{\text {mol }} t}\right] d x=0
$$

Et si l'on défini la moyenne quadratique de l'ensemble des positions par:

$$
\left\langle x^{2}\right\rangle=\int_{-\infty}^{+\infty} x^{2} \Phi(x, t) d x
$$

En se servant de l'intégrale: $\int_{-\infty}^{+\infty} y^{2} \exp \left(-y^{2} d y\right)=\sqrt{\frac{\pi}{4}}$ et en effectuant le changement $y^{2}=\frac{x^{2}}{4 D t}$, l'équation (10) devient: $\left\langle x^{2}\right\rangle=2 D_{\text {mol }} t$

Sachant que $\left\langle u_{i}(\mathbf{x}(\mathbf{t}))\right\rangle=0$ et en définissant la corrélation lagrangienne des vitesses par: $C_{i j}(t) \equiv\left\langle u_{i}(\mathbf{x}(\mathbf{t})) u_{j}(\mathbf{x}(\mathbf{0}))\right\rangle$, on obtient:

$$
\begin{aligned}
\left\langle\left(x_{i}(t)-x_{i}(0)\right)^{2}\right\rangle & =2 D_{m o l} t+\int_{0}^{t} \int_{0}^{t}\left\langle u_{i}(\mathbf{x}(\mathbf{t})) u_{j}(\mathbf{x}(0))\right\rangle d t_{1} d t_{2}, \\
& =2 D_{m o l} t+2 \int_{0}^{t} d t_{2} \int_{0}^{t_{2}} C_{i j}\left(t_{2}-t_{1}\right) d t_{1}
\end{aligned}
$$


Pour un temps très grand $(t \rightarrow \infty)$, si la corrélation $\mathrm{Cij}(\mathrm{t})$ se désintègre de manière suffisamment rapide, l'intégrale de l'équation (12) converge alors vers une valeur asymptotique:

$$
\int_{0}^{\infty} C_{i i}(t) d t=\left\langle u_{i}^{2}\right\rangle T_{L}
$$

Où TL définit le temps lagrangien de corrélation.

Donc, en utilisant les équations (12) et (13), on obtient:

$$
\left\langle\left(x_{i}(t)-x_{i}(0)\right)^{2}\right\rangle \approx 2\left(D_{\text {mol }}+\left\langle u_{i}^{2}\right\rangle T_{L}\right) t \equiv 2 D_{i i}^{E} t
$$

Où $\mathrm{D}^{\mathrm{E}}$ ii définit la diffusion effective. Ainsi, à long terme, l'équation (1) peut s'écrire:

$$
\frac{\partial\langle\phi\rangle}{\partial t}=\sum_{i, j} D_{i j}^{E} \frac{\partial^{2}\langle\phi\rangle}{\partial x_{i} \partial x_{j}}
$$

On note que l'équation (14) peut s'écrire encore sous la forme asymptotique suivante:

$$
\left\langle\left(x_{i}(t)-x_{i}(0)\right)^{2}\right\rangle \sim t^{\alpha}, \alpha \neq 1
$$

Lorsque $\alpha \succ 1$, on dit qu'il y a processus de superdiffusion.

\subsection{Equation de Langevin et diffusion effective}

Pour illustrer l'influence de l'évolution temporelle de la perturbation $\sqrt{2 D_{\text {mol }}} \eta(t)$, on considère alors l'équation de Langevin suivante:

$$
\frac{d \mathbf{x}}{d t}=\mathbf{u}(\mathbf{x}(t ; x), t)+\sqrt{2 D_{m o l}} \eta(t), \mathbf{x}(0 ; x)=x
$$

Où $\mathrm{x}(\mathrm{t})=(\mathrm{x}(\mathrm{t}), \mathrm{y}(\mathrm{t}))$ définit la trajectoire lagrangienne; $\mathrm{u}=(\mathrm{u}, \mathrm{v})$ le vecteur vitesse bidimensionnelle qui caractérise la partie déterministe de l'écoulement et $\sqrt{2 D_{\text {mol }} l}(t)$ sa partie stochastique qui caractérise une perturbation temporelle où mol est la diffusivité moléculaire, ( $\mathrm{t}$ ) vérifie l'équation de corrélation suivante: $\left\langle\eta_{i}(t) \eta_{j}\left(t^{\prime}\right)\right\rangle=\delta_{i j} \delta\left(t-t^{\prime}\right)$ est la fonction de Dirac, et $\langle\rangle=.\frac{1}{N} \sum_{k=1}^{N}($.

A partir des déplacements lagrangiens obtenus des équations (14) et (17), la diffusion effective ${ }^{D_{i j}^{E}}$,s'écrit [2]:

$$
D_{i j}^{E}=\lim _{t \rightarrow \infty} \frac{1}{2 t}\left\langle\left(x_{i}(t)-\left\langle x_{i}(t)\right\rangle\right)\left(x_{j}(t)-\left\langle x_{j}(t)\right\rangle\right)\right\rangle \quad i, j=1,2
$$

Cette diffusion tient compte alors de tous les processus présents dans l'écoulement, à savoir la turbulence, les processus aléatoires, de la présence ou non de structures tourbillonaires, etc. 


\section{Applications}

\subsection{Cas académique}

Dans la suite de ce travail, on examinera le cas simple d'un champ bidimensionnel staionnaire de vitesse périodique $\mathbf{u}_{s}=(u, v)=[B \cos (k y), A \sin (k x)]$ (voirFigures1), auquel on a ajouté une perturbation temporelle périodique: $\eta(t)=(\cos (2 \pi t), \cos (2 \pi t))$

L'équation (17) devient:

$$
u=B \cos (k y)+\varepsilon \cos (2 \pi t) ; v=A \sin (k x)+\varepsilon \cos (2 \pi t)
$$

Où $\mathrm{A}$ et $\mathrm{B}$ désignent les amplitudes des vitesses et $\mathrm{k}=2 \pi /$ est le nombre d'onde.

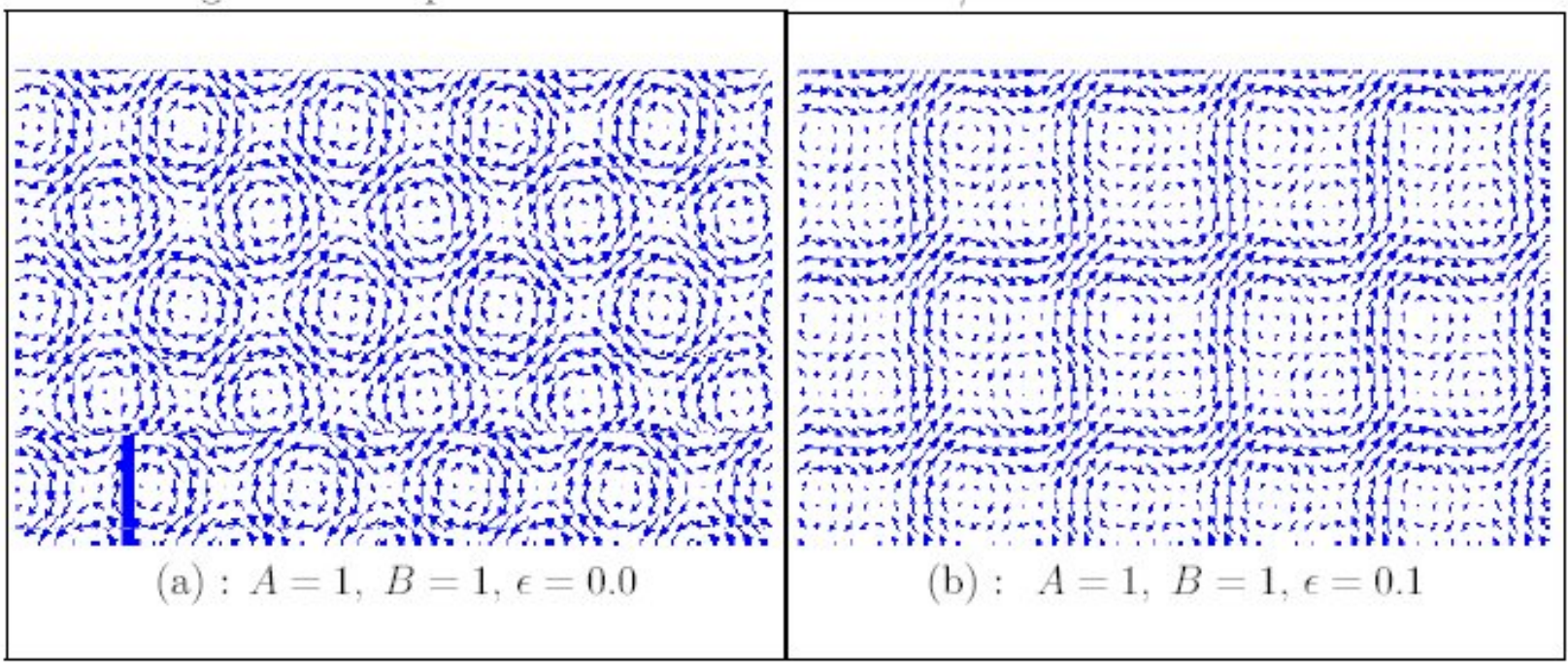

Figure1: Champ des vitesses correspondant à l'équation (19)

\subsubsection{Etude de la stabilité de la solution}

Les solutions stationnaires du problème (17) sans la perturbation temporelle vérifient [?]:

$$
k x_{s}=p \pi ; k y_{s}=(2 n+1) \frac{\pi}{2} \text { avec } p, n \in \Re
$$

Si l'on tient compte de la perurbation (t), les trajectoires subissent des déviations $\widetilde{x}(t)$ et $\widetilde{y}(t)$ suivant les directions $\mathrm{x}$ et $\mathrm{y}$, telles que:

$$
x=x_{s}+\widetilde{x}(t) \text { et } y=y_{s}+\widetilde{y}(t)
$$

Ainsi du système (17) on obtient:

$$
\begin{aligned}
& \frac{d^{2} \widetilde{x}(t)}{d d^{2}}+k^{2} A B(-1)^{n+p} \widetilde{x}(t)=0 \\
& \frac{d^{2} \widetilde{y}(t)}{d t^{2}}+k^{2} A B(-1)^{n+p} \widetilde{y}(t)=0
\end{aligned}
$$


A ce stade d'analyse, il est nécessaire d'examiner l'évolution temporelle des déviations ${ }^{\widetilde{x}(t)}$ et $\widetilde{y}(t)$ lorsqu'on s'éloigne de la position stationnaire (xs,ys). Ainsi, en tenant compte des conditions initiales $(\mathrm{x} 0, \mathrm{y} 0)$ et en considérant le cas $(\mathrm{AB}>0)$ avec $\mathrm{m}=\mathrm{n}+\mathrm{p}$, deux cas se présentent:

cas1: $m=2 p$

Du système (22) on obtient deux solutions stables (Fig.2), bornées et périodiques, qui vérifient:

$$
\begin{aligned}
& \widetilde{x}(t)=x_{0}[\exp (+i k \sqrt{A B} t)+\exp (-i k \sqrt{A B} t)] \\
& \widetilde{y}(t)=y_{0}[\exp (+i k \sqrt{A B} t)+\exp (-i k \sqrt{A B} t)]
\end{aligned}
$$

cas2: $m=2 p+1$

Du système (22) on obtient deux solutions apériodiques dont l'une s'attenue au cours du temps et l'autre croît et correspond à la solution instable (Fig.2). Elles vérifient:

$$
\begin{aligned}
& \widetilde{x}(t)=x_{0}[\exp (+k \sqrt{A B} t)+\exp (-k \sqrt{A B} t)] \\
& \widetilde{y}(t)=y_{0}[\exp (+k \sqrt{A B} t)+\exp (-k \sqrt{A B} t)]
\end{aligned}
$$

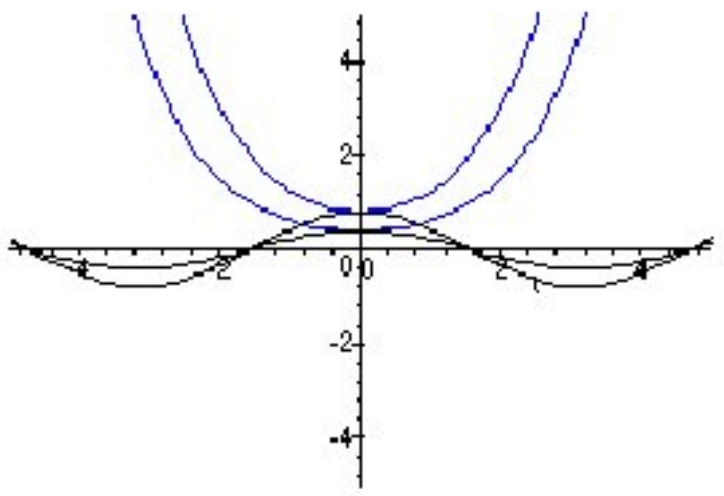

Figure 2: Solutions stables (ligne continue) et instables (ligne pointillée). $(x 0, y 0)=(0.4,0.2)$

\subsubsection{Simulation numérique.}

Dans cette simulation numérique, on a examiné les trajectoires lagrangiennes d'un nuage de 400 particules lâchées dans l'écoulement défini par l'équation (19). On note que $=0$ correspond au cas où les lignes de courants ont toutes fermées ce qui correspond à la présence exclusive de tourbillons, tandis que $=0.1$ correspond au cas où les lignes de courant ne sont pas toutes fermées et que des tourbillons et des allées coexistent. Sur les figures3-a et 3-b sont présentées les variations des coefficients de diffusion effective longitudinal D11 et transversal D22 en fonction du temps. Ces coefficients sont définis à partir de l'équation (18) par:

$$
D_{11}=\lim _{t \rightarrow \infty} \frac{1}{2 t}\left\langle(x(t)-\langle x(0)\rangle)^{2}\right\rangle ; \quad D_{22}=\lim _{t \rightarrow \infty} \frac{1}{2 t}\left\langle(y(t)-\langle y(0)\rangle)^{2}\right\rangle
$$



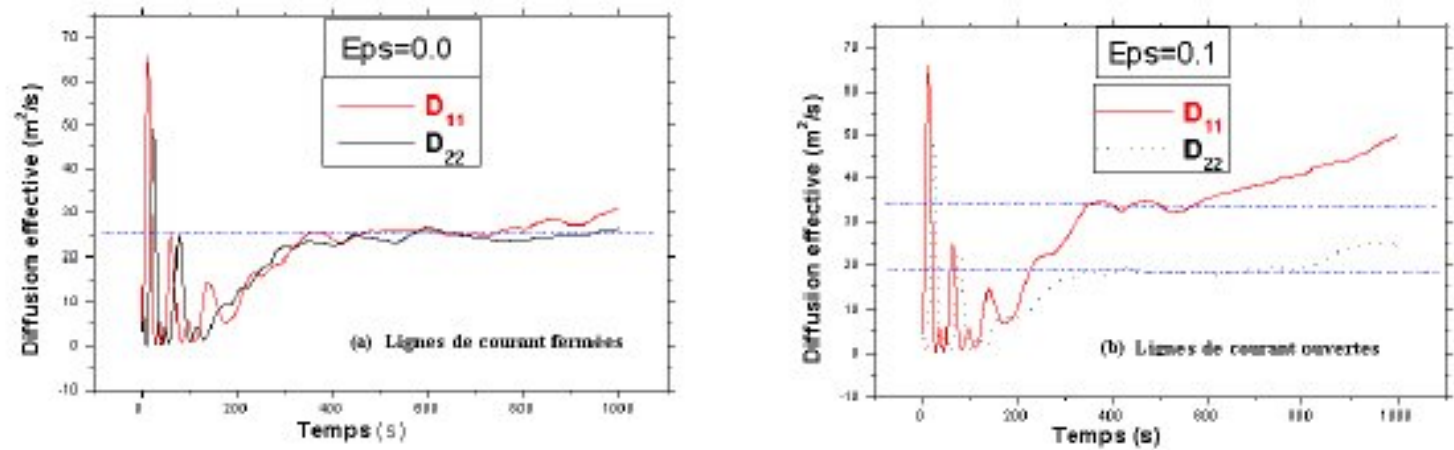

Figure 3: Variation de la diffusion effective D11 et D22 en fonction du temps.
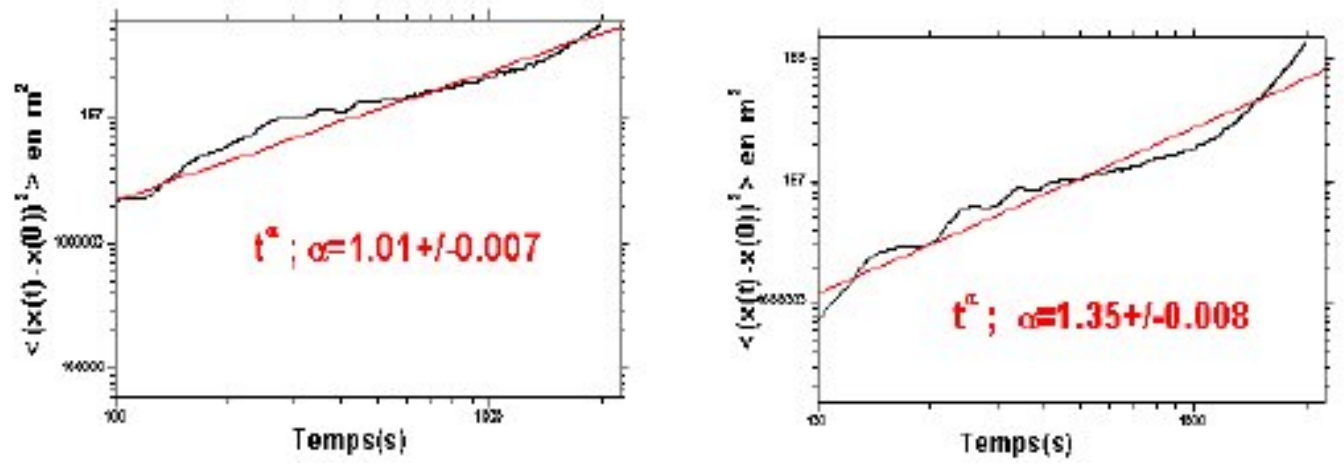

Figure 4: Variation quadratique des trajectoires en fonction du temps.

\subsubsection{Cas réel}

Les simulations sont réalisées ici par un modèle hydrodynamique $2 \mathrm{~d}[5]$ conçu pour étudier la dynamique des courants de marée dans la Manche Orientale. Ce modèle résout les équations de Navier-Stokes tridimensionnelles intégrées suivant une verticale, dont les équations de continuité, de quantité de mouvement et de concentration, sont données par:

$$
\begin{aligned}
\partial \eta_{t}+\nabla \cdot(H \mathbf{v}) & =0 \\
\partial(H \mathbf{v})_{t}+\nabla(H \mathbf{v}) \cdot \mathbf{v}+f \mathbf{k} \wedge(H \mathbf{v}) & =-g H \nabla \xi+\nabla \cdot\left(A_{h} \nabla(H \mathbf{v})\right)-\tau_{b /} \varrho_{0} \\
\partial(H \mathbf{C})_{t}+\nabla(H \mathbf{C}) \cdot \mathbf{v} & =\nabla \cdot\left(A_{h} \nabla(H C)\right)
\end{aligned}
$$

Où $\mathbf{v}=\frac{1}{H} \int_{-z_{b}}^{\eta}(u, v) d z$ est le vecteur vitesse horizontale intégrée suivant une verticale,

$$
\nabla=(\partial / \partial x, \partial / \partial y) ; H=\eta+h \text { est la profondeur totale avec est la dénivellation }
$$
de la surface libre et h est la profondeur, g est l'accélération de la pesanteur; est la densité de l'eau; C'est une concentration passive, $\mathrm{f}=2 \Omega$ sin est le paramètre local de Coriolis; A h est le coefficient de viscosité turbulente horizontale, supposée constante; $\tau_{b}\left(\tau_{b}^{x}, \tau_{b}^{y}\right)=\frac{\rho g}{C_{h}^{2}}\|\mathbf{v}\| \mathbf{v}$ désigne le vecteur contraintes au fond (Ch'77.50m s $\mathrm{s}^{1 / 2}$ ). Aux équations (25)-(27) sont associées 
des conditions aux limites et initiales (voir[5]). La figure 5 représente les lignes de courants assoociées à la circulation résiduelle eulérienne. La figure 6 montre la dispersion d'un nuage de particules lachées dans un tourbillon se trouvant au milieu de cette zone. Après 27 heures de calculs une partie de ces particules reste piegée au niveau de ce tourbillon, avec un temps de résidence assez grand. Après avoir quitté cette zone, elles sont très rapidement dispersées et transportées par les courants.

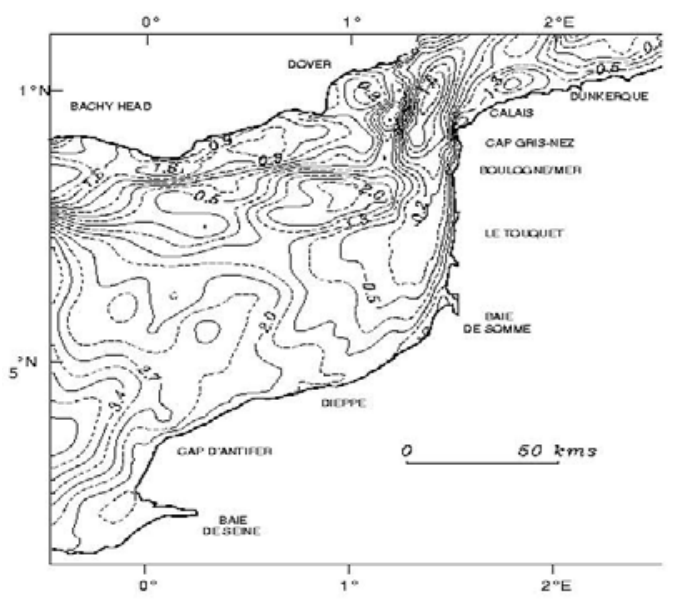

Figure 5: Lignes de courant du champ moyen des vitesses. (Ouahsineetal)

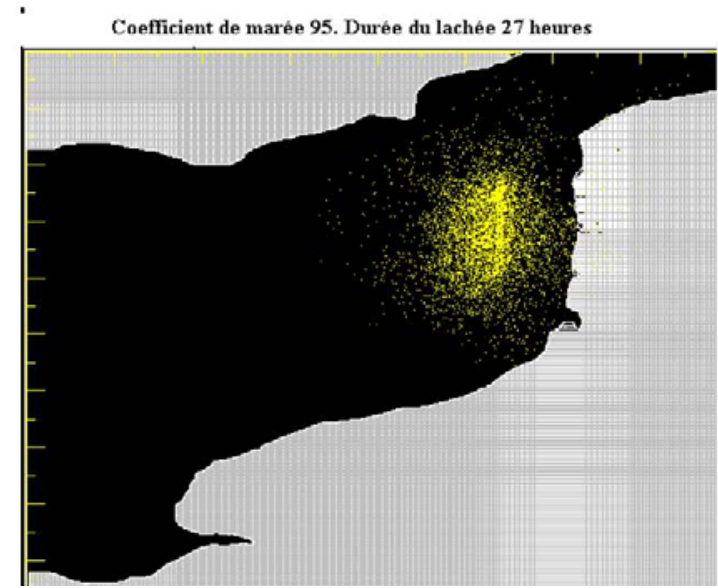

Figure 6: Lâchée d'un nuage de 500 particules dans ce champs moyen.

\section{Conclusion}

Dans ce travail, il a été étudié la diffusion effective en examinant les trajectoires lagrangiennes d'un nuage de particules. Ces trajectoires sont obtenues à partir de l'équation de Langevin en tenant compte d'une perturbation supposée dépendre du coefficient de dif-fusivité moléculaire. Les résultats montrent que cette diffusion dépend de la nature du champs des vitesses et de l'ordre de grandeur du coefficient de diffusion moléculaire. Une loi en puissance est alors proposée suivant la nature de l'écoulement.

\section{Références}

1.Stremler,M.A.,Aref,H., 1999 Motion of three vortices in a periodic parallelogram. Journal of Fluids Mechanics. 392, 101-128.

2.Artale, V., Boffetta, G.,Celani, A.,Cencini, M.,Vulpiani, A., 1997 Dispersion of passive tracers in closed basins: beyond the diffusion coefficient, Phys.Fluids 9, 3162.

3.Korotenko,K.A.. Matter Transportin Mesoscale Oceanic Fronts of River Discharge Type, Journal of Marine Systems 24 (2000) 85-95

4.Weiss J.B., A.Provenzale, J.C. Mcwilliams, Lagrangian dynamics in high-dimensional point-vortex systems, Phys. Fluids 108 (1998)

5.Ouahsine A., Smaoui H, (1999), Flux-limiter schemes for oceanic tracers: Application to the English Channel tidal model .J. Comp. Meth. Appl. Mechand Eng. 179, 307-325. 